\title{
Nutrition, movement and sleep behaviours: their interactions in pathways to obesity and cardiometabolic diseases
}

\author{
A. G. Dulloo, J. L. Miles-Chan and J.-P. Montani
}

${ }^{1}$ Department of Medicine, Division of Physiology, University of Fribourg, Fribourg, Switzerland

Address for correspondence: Prof. Abdul G Dulloo, Department of Medicine, Division of Physiology, University of Fribourg, Chemin du Musée 5, CH 1700 Fribourg, Switzerland. E-mail: abdul.dulloo@unifr.ch

\begin{abstract}
Summary
Among the multitude of dietary and lifestyle behaviours that have been proposed to contribute to the obesity epidemic, those that have generated considerable research scrutiny in the past decade are centred upon sleep behaviours, sedentary behaviours (sitting or lying while awake) and diminished low-level physical activities of everyday life, with each category of behaviours apparently presenting an independent risk for obesity and/or cardiometabolic diseases. These behaviours are highly complex, operate in synergy with each other, disrupt the link between regulation of the circadian clock and metabolic physiology and impact on various components of daily energy expenditure and feeding behaviours to promote obesity and hinder the outcome of obesity therapy. As such, this behavioural triad (nutrition, movement and sleep) presents plenty of scope for intervention and optimization in the context of body weight regulation and lifestyle-related disease prevention. It is against this background that recent advances relevant to the theme of 'Nutrition, Movement \& Sleep Behaviors: their interactions in pathways to obesity and cardiometabolic diseases' are addressed in this overview and the nine review articles in this supplement reporting the proceedings of the 8th Fribourg Obesity Research Conference.
\end{abstract}

Keywords: Energy balance, energy expenditure, physical activity, sedentary.

\section{Introduction}

The 8th Fribourg Obesity Research Conference, which was attended by researchers from both academia and industry, provided a platform for scientists in both basic science and clinical medicine to present and discuss some of the rapidly advancing scientific findings pertaining obesity and metabolic health from a perspective of integrative physiology and nutrition. Its proceedings consist of a total of nine review articles which, together with abstracts of poster sessions, are published in this supplement. An overview of the topics and main issues addressed at this conference and in the review articles is provided in the succeeding texts.

\section{Behavioural clusters and circadian disruption in obesity predisposition and management}

Traditionally, the importance of physical activity in the development of obesity has centred upon a lack of moderate-to-vigorous physical activity. During the past 15 years, however, a large body of evidence has also pin-pointed the potential importance of a lack of low-level physical activity of everyday life and insufficient sleep in the pathogenesis of obesity and chronic metabolic diseases $(1,2)$. Indeed, sedentary behaviour - in particular too much sitting or lying when awake - is now recognized as an independent risk factor for obesity and cardiometabolic diseases (3), and that the associations between prolonged sitting and health risks 
are independent of time spent in moderate-to-vigorous physical activity (4-6).

In the opening article of this supplement, Chaput et al. (7), after reviewing studies that have addressed the effects of physical activity, sedentary and sleep behaviours on health outcomes in children, emphasize that (i) combinations of behaviours may affect health in ways that cannot be explained by the effect of individual behaviours studied in isolation, and that (ii) the clustering of interactions among sleep, movement and sedentary behaviours suggests that all components of the 24-h movement continuum should be targeted to maximize health benefits and prevent childhood obesity.

They also underscore the fact that when it comes to examining sleep as a risk factor for obesity, the question of what is optimal sleep goes well beyond the notion of sleep quantity or duration and includes dimensions such as sleep quality (i.e. efficiency of staying asleep), timing (i.e. bedtime/wake up time), architecture (i.e. sleep stages), consistency (day-to-day variability) and continuity (variability in sleep duration within the same night). In particular, as underscored by McHill and Wright (8), sleep disruption and circadian misalignment (when daily routines such as wakefulness and food intake occur when our internal circadian clock is promoting sleep) are now recognized as novel risk factors that contribute to dysregulated metabolic physiology. After examining the relationship between both sleep and circadian physiology with energy metabolism, they highlight the evidence as to how and why disruption of sleep and circadian physiology contributes to chronic metabolic diseases, a link which has wide-reaching implications in our modern society where shift-working and late-night social activities are increasingly becoming the norm.

The molecular mechanisms implicated in this link between short sleep duration and circadian disruption with higher risk for obesity and the metabolic syndrome are then explored by Albrecht (9). After introducing the dynamic organization of circadian metabolism which is controlled by three oscillating systemic networks working in synchrony (light-entrainable central oscillations in the brain, sleep/ wake homeostasis and feeding/fasting rhythms), he discusses how de-synchronization due to alteration of one of the networks leads to impaired metabolic regulation and the development of chronic metabolic diseases, with obesity and diabetes as the most prominent manifestations. More specifically, he discusses how metabolites and nutrients regulate the circadian clock, how nuclear receptors can act as metabolic sensors and clock regulators and how clock modulation and feeding patterns influence the development of obesity. As the gut microbiome displays cyclic changes according to the feeding/fasting rhythms, the issue is also raised as to whether and how the gut microbiome may affect local and systemic rhythms in metabolism and physiology and could further contribute to the development of obesity and metabolic disease.
From a clinical research perspective, however, the causal mechanisms linking short sleep duration and obesity remain uncertain, amid several proposals for mechanisms that operate via altered energy intake or energy expenditure. In her appraisal of the 'sleep-obesity relation', St Onge (10) discusses the outcome of clinical interventions that have explored these various proposals, namely (i) whether the extra time awake provides more opportunity to eat; (ii) whether short sleep results in increased hunger from hormones that signal appetite and/or reduced satiety from hormones that promote satiation; and (iii) whether increased fatigue related to short sleep results in lower physical activity level. In addition, she provides an analysis of whether weight loss is hindered when attempted in the context of sleep restriction and the feasibility of sleep extension in short sleepers as a strategy for improving the prognosis of weight management.

\section{Post-exercise behavioural compensations}

With the recognition that sleep, sedentary behaviour and low-level physical activity together make up most ( 95\%) of the day for an increasingly large section of the population, it is clear that focusing exclusively on promoting moderate-to-vigorous physical activity (which accounts for $\sim 5 \%$ of the day) limits the potential to optimize the health benefits of movement behaviours. Furthermore, it is a common observation that regular, structured exercise often leads to a less than expected decrease in body weight, thereby suggesting that either there is compensatory increases in energy intake or that some compensatory behavioural mechanisms operate to diminish post-exercise physical activity. In other words, the question arises as whether the adoption of regular, structured exercise leads to compensatory changes in behaviour and/or physiology that may attenuate the expected increases in total daily energy expenditure. These issues are addressed by Melanson (11) in his analysis of human intervention studies that have investigated the effect of exercise on non-exercise physical activity and sedentary behaviour in adults. He subsequently discusses some of the challenges and methodological considerations associated with measuring changes in behaviour and physiology and proposes some frameworks which could guide study design to better address how structured exercise impacts on energy expenditure in humans.

\section{Smart monitoring of physical activity behaviours}

The monitoring of habitual physical activity behaviours, however, is a challenging task. Amid the numerous activity monitors described in the scientific literature and commercial devices appearing on the market at unprecedented rates, an important issue is to decide what type of wearable sensors can be applied to best objectively assess daily life energy 
expenditure and determine how activity recognition may help to improve this assessment. As Plasqui (12) emphasizes in his review on 'smart' approaches for assessing free-living energy expenditure, such monitoring ideally requires validated techniques that can (i) reliably measure physical activity and all its different components (intensity, duration and type) under the unrestrained conditions of daily life, and (ii) that can be employed to stimulate physical activity by objective and continuous monitoring and feedback on activity behaviour. While providing an overview of the available technology, with a special focus on activity recognition, he also discusses the limitations for such energy expenditure assessment under truly free-living conditions.

\section{Neglected sources of skeletal muscle thermogenesis}

In the context of activity recognition, one facet of physical activity of daily life that is rarely considered in relation to energy balance is the role of heat production due to isometric work and hence muscle tension (i.e. isometric thermogenesis). While most physical activities consist of both dynamic and static (isometric) muscle actions, the isometric component is very often essential for the optimal performance of the dynamic work given its role in the coordination of posture during standing, walking and most physical activities of everyday life. In their review on isometric thermogenesis in everyday life, Dulloo et al. (13) bring forward evidence suggesting that this often overlooked component of energy expenditure may have relevance to both energy balance and thermal balance, and in particular in relation to (i) a role for skeletal muscle minor tremor or microvibration - nowadays referred as 'resting muscle mechanical activity' - in maintaining body temperature in response to mild cooling; (ii) a role for slowed skeletal muscle isometric contraction-relaxation cycle as a mechanism for energy conservation in response to caloric restriction and weight loss; and (iii) a role for spontaneous physical activity - which is contributed importantly by isometric work for posture maintenance and fidgeting behaviours - in adaptive thermogenesis pertaining to weight regulation. They review the evidence of these proposed roles for isometric work in adaptive thermogenesis and subsequently put forward the case that variability in this neglected component of energy expenditure could contribute to human predisposition to obesity.

\section{Adipose tissue browning and brown adipose tissue thermogenesis}

Given the difficulties of implementing healthy behaviours in our obesogenic environment, the notion of 'exercise in a pill' by targeting brown adipose tissue (BAT) thermogenesis remains an attractive approach for obesity management.
This avenue for research has, over the past decade, received considerable reinforcement following the realization that BAT can be activated or recruited through the browning of white adipose tissue in adult humans (14). Interestingly, there is emerging evidence that exercise training may also promote the browning of white adipose tissue $(15,16)$, with various theories of the underlying mechanisms centred upon the effect of exercise in increasing sympathetic activity, the secretion of hypothalamic brain-derived neurotrophic factor or upon the secretion of various myokines released from skeletal muscle during exercise, including irisin, Interleukin 6 , meteorin-like protein and $\beta$-aminoisobutyric acid. This area of research is in its very early stage, amid numerous questions including about the potential contribution of different exercise types and exercise intensity in adipose tissue browning and in the activation and recruitment of BAT thermogenesis to combat obesity and cardiometabolic diseases.

What is now evident is that brown adipocytes regulate energy expenditure via mitochondrial uncoupling across a highly complex network underlying brown fat cell differentiation and cellular machinery leading to thermogenic activation. In their review on this topic, Pradhan et al. (17) present a systems perspective on brown adipogenesis and the subsequent metabolic activation of brown adipocytes by integrating signalling, metabolic and gene regulatory modules with a specific focus on known 'druggable' targets within each module. The latter include additional regulators that are capable of inducing browning in concert with key molecular players, thereby providing alternative points of safe therapeutic intervention. While this approach is geared towards the development of 'exercise in a pill' as an antidote to increasing sedentary behaviours and sub-optimal physical activity levels, a better understanding of the mechanisms by which exercise leads to browning of white adipose tissue may well offer powerful insights in search for such druggable targets.

\section{Tackling obesity at the community and societal levels}

From a public health standpoint, however, any success in obesity management is unlikely to occur without actions at a community level that would ultimately impact energy balance. In addressing the challenges for tackling obesity at the community level by integrating healthy diet, movement and non-movement behaviours, Tremblay and Lachance (18) draw a list of criteria that deserve attention in the global and societal management of obesity, namely (i) the realization that obesity is partly the consequence of subtle changes in our lifestyle such as the undesirable sedentary behaviours and sleep habits characterized by reduced sleep duration and quality; (ii) the development of solutions being accessible to those who need them most, e.g. 
individuals with a low socioeconomic status; (iii) a better use of the school environment to promote healthy behaviours; (iv) to change cosmetic standards of communities including the reduction of the obsession for leanness, a risk for later obesity and cardiometabolic diseases (19-21); and (v) for communities to consider sustainable development. They argue that because most of the environmental deteriorations that are potentially involved in the obesity epidemic are the result of human activities aimed at industrial productivity, it is likely that this issue will put mankind in conflict with itself by opposing money-making preoccupations and human development.

\section{Concluding remarks}

Increasing our levels of moderate-to-vigorous physical activity is an unpractical, unrealistic and/or unattractive means to curb obesity development for a large proportion of the population. In contrast, the modification of lifestyle behaviours associated with nutrition, movement and sleep may present a much more feasible target. The reviews contained within this supplement summarize a number of avenues of potential exploitation, from the molecular to the population level, through which these daily-life activities may be optimized in order to better our health and wellbeing. However, they also highlight the complex synergistic nature of these behaviours and the dangers of considering any in isolation - indeed, put simply, being physically active for an hour a day does not give us 'carte blanche' to rest on our laurels for the remaining 23.

\section{Conflict of interest statement}

All authors declare that there is no conflict of interest.

\section{References}

1. Martínez-González MA, Martínez JA, Hu FB, Gibney MJ, Kearney J. Physical inactivity, sedentary lifestyle and obesity in the European Union. Int J Obes Relat Metab Disord. 1999; 23: 1192-1201.

2. Fogelholm M, Kronholm E, Kukkonen-Harjula K, Partonen T, Partinen M, Härmä M. Sleep-related disturbances and physical inactivity are independently associated with obesity in adults. Int J Obes (Lond). 2007; 31: 1713-1721.

3. Thorp AA, Owen N, Neuhaus M, Dunstan DW. Sedentary behaviors and subsequent health outcomes in adults a systematic review of longitudinal studies, 1996-2011. Am J Prev Med 2011; 41: 207-215.
4. Healy GN, Dunstan DW, Salmon J et al. Objectively measured light-intensity physical activity is independently associated with 2-h plasma glucose. Diabetes Care 2007; 30: 1384-1389.

5. Jakes RW, Day NE, Khaw KT et al. Television viewing and low participation in vigorous recreation are independently associated with obesity and markers of cardiovascular disease risk: EPICNorfolk population-based study. Eur J Clin Nutr 2003; 57: 1089-1096.

6. Stamatakis E, Hirani V, Rennie K. Moderate-to-vigorous physical activity and sedentary behaviours in relation to body mass index-defined and waist circumference-defined obesity. $\mathrm{Br} J \mathrm{Nutr}$ 2009; 101: 765-773.

7. Chaput J-P, Saunders TJ, Carson V. Interactions between sleep, movement and other non-movement behaviours in the pathogenesis of childhood obesity. Obes Rev; 2017; 18(Suppl 1): 7-14.

8. McHill AW, Wright KP Jr. Role of sleep and circadian disruption on energy expenditure and in metabolic predisposition to human obesity and metabolic disease. Obes Rev; 2017; 18 (Suppl. 1): 15-24.

9. Albrecht U. The circadian clock, metabolism and obesity. Obes Rev 2017; 18(Suppl 1): 25-33.

10. St Onge M-P. Sleep-obesity relation: underlying mechanisms and consequences for treatment. Obes Rev 2017; 18(Suppl 1): 34-39.

11. Melanson EL. The effect of exercise on non-exercise physical activity and sedentary behavior in adults. Obes Rev; 2017; 18 (Suppl 1): 40-49.

12. Plasqui G. Smart approaches for assessing free-living energy expenditure following identification of types of physical activity. Obes Rev; 2017; 18(Suppl 1): 50-55.

13. Dulloo AG, Miles-Chan JL, Montani JP, Schutz Y. Isometric thermogenesis at rest and during movement: a neglected variable in energy expenditure and obesity predisposition. Obes Rev; 2017; 18(Suppl 1): 56-64.

14. Dulloo AG. Translational issues in targeting brown adipose tissue thermogenesis for human obesity management. Ann N Y Acad Sci 2013; 1302: 1-10.

15. Stanford KI, Middelbeek RJ, Goodyear LJ. Exercise effects on white adipose tissue: Beijing and metabolic adaptations. Diabetes 2015; 64: 2361-2368.

16. De Matteis R, Lucertini F, Guescini M et al. Exercise as a new physiological stimulus for brown adipose tissue activity. Nutr Metab Cardiovasc Dis 2013; 23: 582-590.

17. Pradhan RN, Zachara M, Deplancke B. A systems perspective on brown adipogenesis and metabolic activation. Obes Rev; 2017; 18(Suppl 1): 65-81.

18. Tremblay A, Lachance E. Tackling obesity at the community level by integrating healthy diet, movement and non-movement behaviors. Obes Rev; 2017; 18(Suppl 1): 82-87.

19. Dulloo AG, Montani JP. Pathways from dieting to weight regain, to obesity and to the metabolic syndrome: an overview. Obes Rev 2015; 16(Suppl 1): 1-6.

20. Montani JP, Schutz Y, Dulloo AG. Dieting and weight cycling as risk factors for cardiometabolic diseases: who is really at risk? Obes Rev 2015; 16(Suppl 1): 7-18.

21. Dulloo AG, Jacquet J, Montani JP, Schutz Y. How dieting makes the lean fatter: from a perspective of body composition autoregulation through adipostats and proteinstats awaiting discovery. Obes Rev 2015; 16(Suppl 1): 25-35. 